\title{
Dreamlet: A New Representation and Migration of Seismic Wavefield in Full Local Domains
}

\author{
Bangyu $\mathrm{Wu}^{1, *}, \mathrm{Ru}-\mathrm{Shan} \mathrm{Wu}^{2}$, Xiudi Jiang ${ }^{3}$, Wenbo Sun ${ }^{3}$ and \\ Jinghuai Gao ${ }^{1}$ \\ ${ }^{1}$ School of Mathematics and Statistics, Xi'an Jiaotong University, Xi'an 710049, \\ P.R. China. \\ ${ }^{2}$ Department of Earth and Planetary Sciences, University of California Santa Cruz, \\ Santa Cruz 95064, USA. \\ ${ }^{3}$ CNOOC Research Institute Co., Ltd., Beijing 100028, P.R. China.
}

Received 7 December 2017; Accepted (in revised version) 4 October 2018

\begin{abstract}
Seismic events have limited time duration, vary with space/traveltime and interact with the local subsurface medium during propagation. Partitioning is a valuable strategy for nonstationary seismic data analysis, processing and wave propagation. It has the potential for sparse data representation, flexible data operation and highly accurate local wave propagation. Various local transforms are powerful tools for seismic data segmentation and representation. In this paper, a detailed description of a multi-dimensional local harmonic transformed domain wave propagation and imaging method is given. Using a tensor product of a Local Exponential Frame (LEF) vector as the time-frequency atom (a drumbeat) and a Local Cosine Basis (LCB) function as the space-wavenumber atom (a beamlet), we construct a time-frequencyspace-wavenumber local atom-dreamlet, which is a combination of drumbeat and beamlet. The dreamlet atoms have limited spatial extension and temporal duration and constitute a complete set of frames, termed as dreamlet frames, to decompose and represent the wavefield. The dreamlet transform first partitions the wavefields using time-space supporting functions and then the data in each time-space blocks is represented by local harmonic bases. The transformed wavefield is downward-continued by the dreamlet propagator, which is the dreamlet atom evolution weightings deduced from the phase-shift one-way propagator. The dreamlet imaging method is formulated with a local background propagator for large-scale medium propagation and combined with a local phase-screen correction for small-scale perturbations. The features of dreamlet migration and imaging include sparse seismic data representation, accurate wave propagation and the flexibility of localized time operations during migration. Numerical tests using Sigsbee 2A synthetic data set and real marine seismic data demonstrate the validity and accuracy of this method. With time-domain localization being involved, the dreamlet method can also be applied effectively to target-oriented migration and imaging.
\end{abstract}

*Corresponding author. Email addresses: bangyuwu@xjtu.edu.cn (B. Wu), rwu@ucsc.edu (R.-S. Wu), jiangxd2@cnooc.com.cn (X. Jiang), sunwb3@cnooc.com.cn (W. Sun), jhgao@mail.xjtu.edu.cn (J. Gao) 
AMS subject classifications: 86A15, 65T60, 34M35

Key words: Seismic imaging, wave equation, local harmonic transform, seismic migration, dreamlet transform.

\section{Introduction}

In practice, the event in seismic section is not stationary, i.e., it changes with offsets and traveltimes. It also has limited time duration and interacts with the local subsurface medium during propagation. The linearity of the seismic events can only be approximated locally in the time space seismogram. For the seismic data compression, localized basis can represent the seismic signals sparsely while still preserving useful information [1-4].

During last decade, wave equation based localized wave propagation and imaging is developed to overcome the limitations of many global migration methods. In strong contrast medium, the wave-equation-based migration methods are accurate, stable and yield results of high quality compared to the algorithms based on eikonal equation solvers, such as Kirchhoff migration [5]. The phase-shift method [6] is accurate, economical and unconditionally stable for media with vertical velocity gradient. Split-Step Fourier (SSF) migration [7] can handle velocity with smooth lateral variations and small lateral contrast. Various phase-screen methods [8-11] extend the concepts of the phase-shift and SSF methods. In such methods, to take lateral velocity variations into account, the medium is decomposed at each level into a global reference velocity and perturbations. For strong contrast media, the perturbations can be very large, leading to difficulties in correctly propagating large-angle waves.

In order to overcome the accuracy limitation for large-angle waves in strong contrast media, several methods based on the local perturbation theory or Locally Homogeneous Approximation (LHA) have been developed. Windowed screen method $[12,13]$ introduces the local background velocity and local perturbations through Windowed Fourier Transform (WFT). However, since the perfect WFT reconstruction is formidably expensive, the method relies on the much broadly overlapped windows and empirical interpolations. Therefore, it is applicable only in the case where only a few distinctive material boundaries exist. Beamlet migration approach based on the local perturbation theory has been proposed using the Gabor-Daubechies Frame (GDF) [14,15] and the Local Cosine Basis (LCB) $[16,17]$. In these methods, the wavefield at every depth is spatially localized with local windows and propagated with beamlet propagators (sparse propagator matrices), followed by local perturbation corrections. Ma and Margrave [18] develops a velocity adaptive partitioning scheme that relates window width to lateral velocity gradient and wider windows are used when the lateral velocity gradient is weak since computation cost is directly proportional to the number of windows. Mousa [19] treats the 
migration as a filtering process and designs short-length finite impulse response filters with both accuracy and efficiency for strong lateral velocity variations.

However, the methods mentioned above only have localization on space and work entirely in frequency domain. The seismic data is nonstationary in nature in both space and time. In order to decompose the wavefields sparsely and efficiently, the elementary function needs to have the ability to adapt to the local variation of the seismic data. Therefore, the frequency domain local wavefield decomposition methods do not offer an impressive compression on seismic data. Efforts have been made to work directly in the compressed domain for seismic migration and imaging for decades. Curvelet transform [20,21], completely localized on time and space, has been successfully applied to seismic data compression [22,23]. Meanwhile, curvelet has also been applied to wave propagation and seismic imaging using a map migration method [24,25]. Also, research has been done to test the effects of using cosine/sine basis to $2 \mathrm{D}$ seismic data compression $[1,26]$, which is proved to be an efficient method providing high compression ratio as well as preserving seismic data information. The dreamlet method [27,28] extends the frequency domain beamlet method to a fully time-space localized wave propagator with time-space local harmonics, applying the background propagation directly in the compressed domain. In this algorithm, the space-wavenumber localization uses the LCB transform and the time-frequency localization adopts the local exponential tight frame $[29,30]$. Localization in time provides seismic processing with another degree of freedom, such as de-noising and local property analysis. Dreamlet migration methods mainly consist four steps: wavefield and propagator decomposition (compression), seismic data migration, reconstruction and local perturbation correction. In the following, we first introduce the local harmonic bases. For the dreamlet migration method, the time-frequency and space-wavenumber localization can be treated separately. Secondly, the detail formulation of the dreamlet propagator based on LCB (space) and LEF (time) decomposition is given. Thirdly, the Sigsbee2A model is first used to demonstrate the validity and image quality of this method on a synthetic model. Making use of time axis localization, target oriented migration and imaging is tested. Field marine seismic data application shows the feasibility and high accuracy of imaging by dreamlet migration for the real data set.

\section{Dreamlet wavefield and one-way propagator decomposition}

\subsection{Local trigonometric bases and local exponential frames}

The basis for local cosine/sine transform is bell function windowed cosine/sine harmonic, also known as local trigonometric basis [31]. For wavefield decomposition on spatial axis, local trigonometric basis can be characterized by position $\bar{x}_{n}$, interval $L_{n}=\bar{x}_{n+1}-\bar{x}_{n}$ (the nominal length of the window function) and wavenumber index $m$ as 
follows

$$
\begin{aligned}
& b_{m n}^{c}(x)=\sqrt{\frac{2}{L_{n}}} B_{n}(x) \cos \left(\pi\left(m+\frac{1}{2}\right) \frac{x-\bar{x}_{n}}{L_{n}}\right), \\
& b_{m n}^{s}(x)=\sqrt{\frac{2}{L_{n}}} B_{n}(x) \sin \left(\pi\left(m+\frac{1}{2}\right) \frac{x-\bar{x}_{n}}{L_{n}}\right),
\end{aligned}
$$

where $B_{n}(x)$ is a bell function which is smooth and supported in the compact interval $\left[\bar{x}_{n}-\varepsilon, \bar{x}_{n+1}-\varepsilon^{\prime}\right]$. The left and right overlapping radius for the local trigonometric transform is defined by $\varepsilon$ and $\varepsilon^{\prime}$. The bar "-" on the top of a parameter represents the localized (phase-space) parameter. If the fourier transform from space to wavenumber is defined as following:

$$
u(\xi)=\int u(x) e^{-i \xi x} d x
$$

we can obtain the wavenumber domain local trigonometric basis

$$
\begin{aligned}
& b_{m n}^{c}(\xi)=\frac{1}{2} \sqrt{\frac{2}{L_{n}}}\left[e^{-i \bar{x}_{n} \bar{\xi}_{m}} B_{n}\left(\xi-\bar{\zeta}_{m}\right)+e^{i \bar{x}_{n} \bar{\zeta}_{m}} B_{n}\left(\xi+\bar{\zeta}_{m}\right)\right] \\
& b_{m n}^{s}(\xi)=\frac{1}{2 i} \sqrt{\frac{2}{L_{n}}}\left[e^{-i \bar{x}_{n} \bar{\zeta}_{m}} B_{n}\left(\xi-\bar{\xi}_{m}\right)-e^{i \bar{x}_{n} \bar{\zeta}_{m}} B_{n}\left(\xi+\bar{\xi}_{m}\right)\right] .
\end{aligned}
$$

In Eqs. (2.4) and (2.5), $B_{n}(\xi)$ is the fourier transform of the bell function $B_{n}(x)$ and $\bar{\xi}_{m}=\pi\left(m+\frac{1}{2}\right) / L_{n}$. It can be seen that the local trigonometric basis always has two symmetric spectral lobes: a positive lobe (centered at $+\bar{\zeta}_{m}$ ) as well as a mirror-symmetric negative lobe (centered at $-\bar{\zeta}_{m}$ ). Therefore the directivity is not uniquely specified for the coefficients of spatial local trigonometric transform. The positive and negative spectral lobes in the trigonometric basis can be separated by the combination of cosine basis function in the real part and sine basis function in the imaginary part, thus forms the local exponential functions [32]:

$$
\begin{aligned}
& g_{m n}^{+}(x)=b_{m n}^{c}(x)+i b_{m n}^{s}(x)=\sqrt{\frac{2}{L_{n}}} e^{i \bar{\xi}_{m}\left(x-\bar{x}_{n}\right)}, \\
& g_{m n}^{-}(x)=b_{m n}^{c}(x)-i b_{m n}^{s}(x)=\sqrt{\frac{2}{L_{n}}} e^{-i \bar{\xi}_{m}\left(x-\bar{x}_{n}\right)}
\end{aligned}
$$

The LEF eliminates the directional ambiguity in the local trigonometric basis and can decompose the wavefield into local wavenumber domain with uniquely defined direction information [30,33]. For spatial decomposition, $g_{m n}^{+}(x)$ and $g_{m n}^{-}(x)$ stand for the right and left propagating beamlets, respectively. Correspondingly, if LEF is used to decompose the time axis, $g_{i j}^{+}(t)$ and $g_{i j}^{-}(t)$ are taken as the positive and negative frequency 
drumbeats,

$$
\begin{aligned}
& g_{i j}^{+}(t)=\sqrt{\frac{2}{T_{j}}} e^{i \bar{\omega}_{i}\left(t-\bar{t}_{j}\right),} \\
& g_{i j}^{-}(t)=\sqrt{\frac{2}{T_{j}}} e^{-i \bar{\omega}_{i}\left(t-\bar{t}_{j}\right)},
\end{aligned}
$$

where $\bar{\omega}_{i}=\pi\left(i+\frac{1}{2}\right) / T_{j}$ is the local frequency, $\bar{t}_{j}$ is the time moment and $T_{j}$ denotes the time interval. The LEF is tight frame with redundancy 2 and the coefficients of a time domain function $f(t)$ can be calculated by the projection

$$
\begin{aligned}
f(t) & =\sum_{i} \sum_{j}\left[\left\langle f(t), g_{i j}^{+}(t)\right\rangle g_{i j}^{+}(t)+\left\langle f(t), g_{i j}^{-}(t)\right\rangle g_{i j}^{-}(t)\right] \\
& =\sum_{i} \sum_{j}\left[f_{i j}^{+} g_{i j}^{+}(t)+f_{i j}^{-} g_{i j}^{-}(t)\right]
\end{aligned}
$$

and $\langle$,$\rangle stands for the inner product. Using the relation between the LEF and LCB/LSB,$ the LEF decomposition coefficients $f_{i j}^{+}$and $f_{i j}^{-}$can be calculated using the LCB and LSB decomposition coefficients $f_{i j}^{c}$ and $f_{i j}^{s}$

$$
\begin{aligned}
& f_{i j}^{+}=\frac{f_{i j}^{c}-i f_{i j}^{s}}{4}, \\
& f_{i j}^{-}=\frac{f_{i j}^{c}+i f_{i j}^{s}}{4} .
\end{aligned}
$$

The LCB and LSB decomposition coefficients can be obtained by fast algorithms [31,34], which makes the LEF decomposition very efficient.

\subsection{Dreamlet wavefield decomposition implementation using LCB and LEF}

A dreamlet (drumbeat-beamlet) atom can be formed by the tensor product of 1D local harmonic atoms, referring to either local cosine/sine basis or local exponential frame vector. The wavefield in the time-space domain or in the frequency-wavenumber domain can be decomposed into the local time-frequency-space-wavenumber domain (dreamlet domain):

$$
\begin{aligned}
& u(x, t)=\sum_{\bar{t}} \sum_{\bar{\omega}} \sum_{\bar{x}} \sum_{\bar{\xi}}\left\langle u, d_{\bar{t} \bar{\omega} \bar{x} \bar{\xi}}(x, t)\right\rangle d_{\bar{t} \bar{\omega} \bar{x} \bar{\xi}}(x, t)=\sum_{\bar{t}} \sum_{\bar{\omega}} \sum_{\bar{x}} \sum_{\bar{\xi}} u_{\bar{t} \bar{\omega} \bar{\omega} \bar{\xi}} d_{\bar{t} \bar{\omega} \bar{x} \bar{\xi}}(x, t) \\
& =\sum_{\bar{t}} \sum_{\bar{\omega}} \sum_{\bar{x}} \sum_{\bar{\xi}} u_{\bar{t} \bar{\omega} \bar{x} \bar{\xi}} g_{\bar{t} \bar{\omega}}(t) b_{\bar{x} \bar{\xi}}(x),
\end{aligned}
$$


where $d_{\bar{t} \bar{\omega} \bar{x} \bar{\xi}}(x, t), g_{\bar{t} \bar{\omega}}(t)$ and $b_{\bar{x} \bar{\xi}}(x)$ are dreamlet, drumbeat and beamlet atom, respectively. And the dreamlet coefficients of the seismic data are obtained

$$
\begin{aligned}
u_{\bar{t} \bar{\omega} \bar{x} \overline{\bar{\zeta}}} & =\left\langle u(x, t), d_{\bar{t} \bar{\omega} \overline{\bar{x}} \overline{\bar{\xi}}}(x, t)\right\rangle=\iint u(x, t) d_{\bar{t} \bar{\omega} \bar{x} \overline{\bar{\zeta}}}^{*}(x, t) d x d t \\
& =\iint u(x, t) g_{\bar{t} \bar{\omega}}^{*}(t) b_{\bar{x} \overline{\bar{c}}}^{*}(x) d x d t .
\end{aligned}
$$

The basis with * is the dual basis. It is flexible to choose any localized bases to achieve the localization in each domain. In this work, we adopt LEF as the temporal and LCB the spatial local decompositions, the dreamlet atoms can be constructed as

$$
d_{\bar{\mu}}(x, t)=g_{i j}^{+}(t) b_{m n}^{c}(x)+g_{i j}^{-}(t) b_{m n}^{c}(x)=d_{\bar{\mu}}^{+}(x, t)+d_{\bar{\mu}}^{-}(x, t),
$$

with $\bar{\mu}=(\bar{t}, \bar{\omega}, \bar{x}, \bar{\xi})$ as the dreamlet local parameter cluster. Since the LCB is orthogonal basis and LEF is tight frame, the dreamlets constructed by the tensor product of LCB and LEF are also frames with redundancy 2 . The dual dreamlet atoms are the same as the original ones. The wavefield decomposition by general dreamlet formulation in Eq. (2.13) can be rewritten with the representation of LEF-LCB dreamlets as

$$
\begin{aligned}
u_{\bar{t} \bar{\omega} \bar{x} \bar{\xi} \bar{\xi}} & =\left\langle u(x, t), d_{\bar{t} \bar{\omega} \bar{x} \overline{\bar{\zeta}}}(x, t)\right\rangle=\iint u(x, t) d_{\bar{t} \bar{\omega} \bar{x} \overline{\bar{\zeta}}}^{*}(x, t) d x d t \\
& =\iint u(x, t) g_{\bar{t} \bar{\omega}}^{*}(t) b_{\bar{x} \bar{\xi}}^{*}(x) d x d t,
\end{aligned}
$$

where $u_{\bar{\mu}}^{+}$and $u_{\bar{\mu}}^{-}$are the dreamlet coefficients for the positive and negative drumbeat frequency. Fig. 1 shows an example of wavefield dreamlet decomposition for the 250th shot of the Sigsbee2A model. Fig. 1(a) is the original seismogram and Fig. 1(b) is the spectrum of the dreamlet coefficients $u_{\bar{\mu}}^{+}\left(u_{\bar{\mu}}^{-}\right.$are conjugate of $\left.u_{\bar{\mu}}^{+}\right)$. In Figs. 1(c), 1(e) and $1(\mathrm{~g})$ are the selected time-space blocks in the seismogram for the detailed demonstration and in Figs. 1(d), 1(f) and 1(h) are the corresponding dreamlet coefficients. We see the sparsity of the dreamlet coefficients of the seismogram. This example shows the capability of dreamlet atoms representing oscillating and directional seismic signals. Dreamlets are decomposed on the data plane and the extrapolated data plane, and in the migration step, only large coefficients are propagated further for subsurface imaging.

\subsection{One-way propagator in the dreamlet domain}

Wu et al. [35] prove that the dreamlet can be considered as a type of physical wavelet defined on an observation plane, which is advantageous for application in wavefield decomposition, propagation and imaging. The dreamlet coefficient of the wavefield can be propagated by ray-based or the one-way wave equation based propagator. Geng et al. [36] give the relationship between dreamlet and Gaussian packet coefficients and the dreamlet coefficients are migrated for long range propagation in smooth heterogeneous 

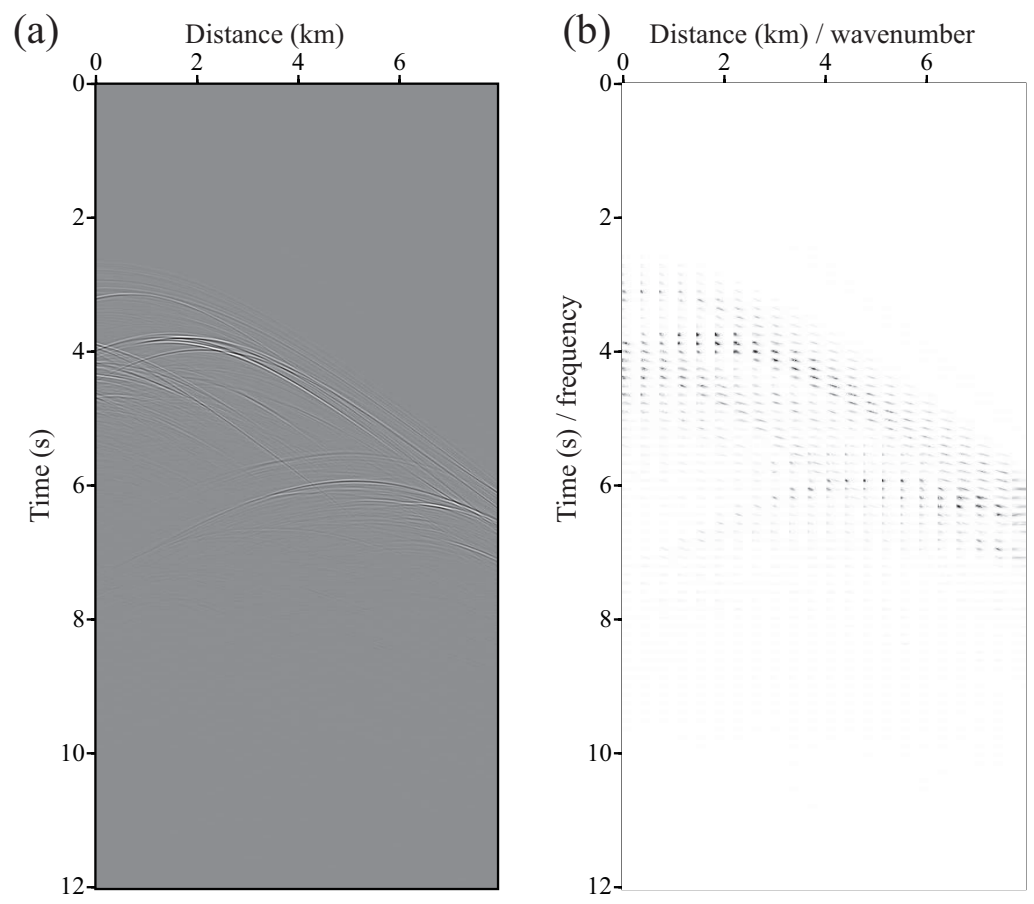

(c)
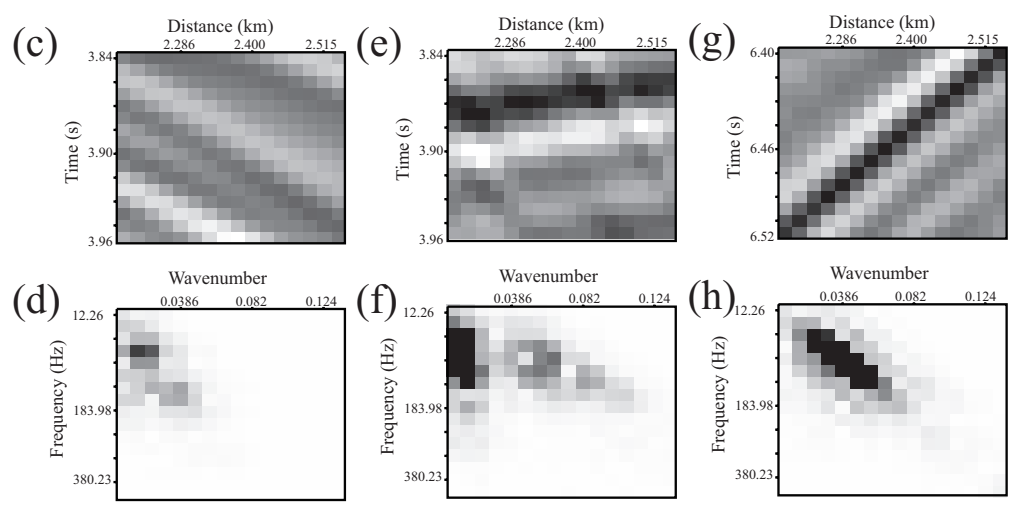

Figure 1: LEF-LCB dreamlet wavefield decomposition of the Sigsbee2A model 250th shot data: (a) seismic data; (b) Dreamlet coefficients; (c), (e) and (g) are the enlargement of the selected window in the seismogram and (d), (f) and (h) below each are their corresponding dreamlet coefficients. Only positive drumbeat coefficients are displayed.

medium by high-frequency asymptotic propagation. The ray based methods can image the steep structures in subsurface but have difficulty in complex areas due to high frequency approximation. The conventional one-way wave equation based migrations downward-continued the wavefield in depth and have limitations on the imaging of challenging structures, such as the overhung salt flanks. However, some recent developed one-way propagators can well image the overhung or vertical structures [37-39]. In this work, we give the dreamlet migration theory based on the one-way wave equation 
migration method. For wave propagation migration and imaging in local phase space, the local decomposition not only decomposes the wavefield but also the propagator to form the phase-space propagator matrix. The main task for the dreamlet migration is to derive propagators in the chosen dreamlet domain. The evolution of dreamlets is governed by an operator which involves a square root operator [40]

$$
a_{\bar{\mu}}(x, t ; \Delta z)=e^{ \pm i \sqrt{\frac{\partial^{2}}{\partial_{x}^{2}}-\frac{1}{v^{2}(x, z)} \frac{\partial^{2}}{\partial_{t}^{2}}} \Delta z} d_{\bar{\mu}}(x, t) .
$$

Where $a_{\bar{\mu}}(x, t ; \Delta z)$ is a function evolved from a dreamlet $d_{\bar{\mu}}(x, t)$ propagating in the heterogeneous medium. The plus and minus sign in Eq. (2.17) are for the downward and upward wave propagation. In this way, the evolution of dreamlets observes the wave equation. After propagation, a dreamlet atom spreads into other cells in the localized phase-space due to propagation distortion. The dreamlet propagator is formulated by the redecomposition of the distorted dreamlet into new dreamlets

$$
P_{\bar{\mu}}^{\bar{\mu}^{\prime}}=\left\langle d_{\bar{\mu}^{\prime}}^{+}+d_{\bar{\mu}^{\prime}}^{-}, a(x, t ; \Delta z)\right\rangle=\left\langle d_{\bar{\mu}^{\prime}}^{+}+d_{\bar{\mu}^{\prime}}^{-} P\left(d_{\bar{\mu}}^{+}+d_{\bar{\mu}}^{-}\right)\right\rangle \text {. }
$$

Here $P=e^{ \pm i \sqrt{\frac{\partial^{2}}{\partial_{x}^{2}}-\frac{1}{v^{2}(x, z)} \frac{\partial^{2}}{\partial_{t}^{2}}} \Delta z}$ stands for the one-way propagator. For the general problem, there is no exact analytical solution for propagator $P$. Various approximations are invoked to make the calculation practical. Wu et al. $[17,41]$ applied local perturbation approximation to the one-way wave equation, resulting in a split-step implementation of wave propagation in the local-phase space domain. The wavefield propagation is stepby-step downward continued using the propagator matrix which incorporates with biscale decomposition of the velocity. At each step, the laterally varying velocity profile is decomposed by window-width piecewise homogeneous medium $v_{0}\left(\bar{x}_{n}, z\right)$ for large scale background propagation and the small-scale component which is the local perturbation. Due to the adaptability of local reference velocity to the lateral variation of the true velocity model, the wave propagation in the local background velocity is accurate enough, so that the first order approximation of the square-root propagator $P$ is good enough for the local perturbation correction. For the large-scale background propagation, dreamlet propagator can be derived by the analytic solution of wave equation in the frequencywavenumber domain for each homogeneous piecewise velocity medium. This leads to the approximation of the one-way operator $P$ as

$$
\begin{aligned}
P & \approx e^{ \pm i \sqrt{\frac{\omega^{2}}{v^{2}\left(\bar{x}_{n}, z\right)}-\tilde{\zeta}^{2}} \Delta z} e^{ \pm i \omega\left(\frac{1}{v(x, z)}-\frac{1}{v\left(\bar{x}_{n}, z\right)}\right) \Delta z} \\
& =e^{ \pm i \zeta_{n} \Delta z} e^{ \pm i \omega\left(\frac{1}{v(x, z)}-\frac{1}{v\left(\bar{x}_{n}, z\right)}\right) \Delta z} \\
& =P^{0}\left(\bar{x}_{n} ; \Delta z\right) P^{1}\left(x, \bar{x}_{n} ; \Delta z\right),
\end{aligned}
$$

where $\zeta_{n}=\sqrt{\omega^{2} / v^{2}\left(\bar{x}_{n}, z\right)-\xi^{2}}$ is the local vertical wavenumber with the local reference velocity $v_{0}\left(\bar{x}_{n}, z\right)$ and $\xi$ is the horizontal wavenumber. Eq. (2.19) is a dual domain splitstep approximation of the operator $P$. The first term $P^{0}\left(\bar{x}_{n} ; \Delta z\right)$ in Eq. (2.19) is a phase 
shift in the local frequency-wavenumber domain using the local reference velocity; the second term $P^{1}\left(x, \bar{x}_{n} ; \Delta z\right)$ is a local phase-screen term in the local frequency-space domain. Here below, we give the dreamlet propagator derivation for the local reference velocity propagation. Eq. (2.17) can be rewritten as

$$
\begin{aligned}
P_{\left(\bar{\mu}^{\prime}, \bar{\mu}\right)}^{0}\left(\bar{x}_{n} ; \Delta z\right)= & \left\langle d_{\bar{\mu}^{\prime},}^{+} P^{0}\left(\bar{x}_{n} ; \Delta z\right) d_{\bar{\mu}}^{+}\right\rangle+\left\langle d_{\bar{\mu}^{\prime}}^{+}, P^{0}\left(\bar{x}_{n} ; \Delta z\right) d_{\bar{\mu}}^{-}\right\rangle \\
& +\left\langle d_{\bar{\mu}^{\prime}}^{-} P^{0}\left(\bar{x}_{n} ; \Delta z\right) d_{\bar{\mu}}^{+}\right\rangle+\left\langle d_{\bar{\mu}^{\prime}}^{-}, P^{0}\left(\bar{x}_{n} ; \Delta z\right) d_{\bar{\mu}}^{-}\right\rangle \\
= & P_{\left(\bar{\mu}^{\prime}, \bar{\mu}\right)}^{0(++)}(\Delta z)+P_{\left(\bar{\mu}^{\prime}, \bar{\mu}\right)}^{0(+)}(\Delta z)+P_{\left(\bar{\mu}^{\prime}, \bar{\mu}\right)}^{0(-)}(\Delta z)+P_{\left(\bar{\mu}^{\prime}, \bar{\mu}\right)}^{0(-)}(\Delta z) .
\end{aligned}
$$

The four components in Eq. (2.20) stand for the different interactions between positive and negative frequencies. Taking $P_{\left(\bar{\mu}^{\prime}, \bar{\mu}\right)}^{0(++)}\left(\bar{x}_{n} ; \Delta z\right)$ for example, insert the time-frequency atoms into the propagator, and then we get

$$
P_{\left(\bar{\mu}^{\prime}, \bar{\mu}\right)}^{0(++)}\left(\bar{x}_{n} ; \Delta z\right)=\frac{1}{2 \pi} \int d \omega\left(g_{\bar{t}^{\prime} \bar{\omega}^{\prime}}^{+}(-\omega)\right)^{*} g_{\bar{t}^{\prime} \bar{\omega}^{\prime}}^{+}(\omega) P_{\left(\bar{x}^{\prime} \bar{\zeta}^{\prime} \bar{x} \bar{\xi}\right)}^{0}(\omega, \Delta z),
$$

where $g_{\bar{t} \bar{\omega}}^{+}(\omega)$ is the fourier transform of $g_{\bar{t} \bar{\omega}}^{+}(t)$ and

$$
P_{\left(\bar{x}^{\prime} \bar{\zeta}^{\prime} \bar{x} \bar{\xi}\right)}^{0}(\omega, \Delta z)=\frac{1}{(2 \pi)^{D-1}} \int d \xi b_{\bar{x}^{\prime} \bar{\xi}^{\prime}}^{*}(-\xi) b_{\bar{x} \bar{\xi}}(\xi) e^{ \pm i \zeta_{n}(\omega, \bar{\xi}) \Delta z}
$$

is the beamlet propagator [17]. In Eq. (2.22), D standing for 2 or 3 is the spatial dimension. For the simplification of migration operation, we keep the bell function same for all the time windows, then

$$
g_{\bar{t} \bar{\omega}}^{+}(\omega)=\sqrt{\frac{2}{T_{j}}} e^{i(\omega+\bar{\omega}) \frac{T}{2}} e^{i \omega \bar{t}} B_{0}(\omega+\bar{\omega}) .
$$

Here $B_{0}(\omega)$ is the fourier transform of time window at $\bar{t}=0$.

Plugging Eq. (2.23) into Eq. (2.21), the dreamlet propagator equation (2.21) becomes

$$
P_{\left(\bar{\mu}^{\prime}, \bar{\mu}\right)}^{0(++)}(\Delta z)=\frac{1}{\pi T} \int d \omega e^{i \omega\left(\bar{t}-\bar{t}^{\prime}\right)} e^{i\left(\bar{\omega}-\bar{\omega}^{\prime}\right) \frac{T}{2}} B_{0}(\omega+\bar{\omega}) B_{0}\left(\omega+\bar{\omega}^{\prime}\right) P_{\left(\bar{x}^{\prime} \bar{\xi}^{\prime} \bar{x} \bar{\xi}\right)}^{0}(\omega, \Delta z) .
$$

In a similar way, other components of the dreamlet propagator can be expressed as

$$
\begin{aligned}
& P_{\left(\bar{\mu}^{\prime}, \bar{\mu}\right)}^{0(+-)}(\Delta z)=\frac{1}{\pi T} \int d \omega e^{i \omega\left(\bar{t}-\bar{t}^{\prime}\right)} e^{i\left(\bar{\omega}+\bar{\omega}^{\prime}\right) \frac{T}{2}} B_{0}(\omega+\bar{\omega}) B_{0}\left(\omega-\bar{\omega}^{\prime}\right) P_{\left(\bar{x}^{\prime} \bar{\xi}^{\prime} \bar{x} \bar{\xi}\right)}^{0}(\omega, \Delta z), \\
& P_{\left(\bar{\mu}^{\prime}, \bar{\mu}\right)}^{0(-+)}(\Delta z)=\frac{1}{\pi T} \int d \omega e^{i \omega\left(\bar{t}-\bar{t}^{\prime}\right)} e^{i\left(-\bar{\omega}-\bar{\omega}^{\prime}\right) \frac{T}{2}} B_{0}(\omega-\bar{\omega}) B_{0}\left(\omega+\bar{\omega}^{\prime}\right) P_{\left(\bar{x}^{\prime} \bar{\zeta}^{\prime} \bar{x} \bar{\xi}\right)}^{0}(\omega, \Delta z), \\
& P_{\left(\bar{\mu}^{\prime}, \bar{\mu}\right)}^{0(--)}(\Delta z)=\frac{1}{\pi T} \int d \omega e^{i \omega\left(\bar{t}-\bar{t}^{\prime}\right)} e^{i\left(-\bar{\omega}-\bar{\omega}^{\prime}\right) \frac{T}{2}} B_{0}(\omega-\bar{\omega}) B_{0}\left(\omega-\bar{\omega}^{\prime}\right) P_{\left(\bar{x}^{\prime} \bar{\zeta}^{\prime} \bar{x} \bar{\xi}\right)}^{0}(\omega, \Delta z) .
\end{aligned}
$$

Here $P_{\left(\bar{\mu}^{\prime}, \bar{\mu}\right)}^{0(++)}, P_{\left(\bar{\mu}^{\prime}, \bar{\mu}\right)}^{0(+-)}, P_{\left(\bar{\mu}^{\prime}, \bar{\mu}\right)}^{0(-+)}$ and $P_{\left(\bar{\mu}^{\prime}, \bar{\mu}\right)}^{0(-)}$ are the kernels of the dreamlet propagator in the background media. The plus and minus sign stand for the positive and negative local 
frequencies respectively. Since the coefficients for the positive and negative frequency drumbeat are conjugated, only two of the propagator matrices in Eqs. (2.24)-(2.27) are needed. In the following tests, we only use $P_{\left(\bar{\mu}^{\prime}, \bar{\mu}\right)}^{(++)}$and $P_{\left(\bar{\mu}^{\prime}, \bar{\mu}\right)}^{0(-+)}$ for positive drumbeat coefficients computation in wave propagation.

\section{Dreamlet prestack depth migration}

The study of single dreamlet atom evolution in space is very important and can be considered as intermediate status of the point source and plane wave. The point source and plane wave are two elementary solutions to the wave equation. The point source solution has exact space location precision but the wave propagates into all directions. The plane wave has unique propagation direction but it extends to infinity spatially. The dreamlet atoms propagate like localized plane wave in the media. In Fig. 2, it shows composite plot of three snapshots of the single dreamlet atom propagation in a homogenous medium. Fig. 2(a) is the snapshots of a vertical propagating dreamlet and Fig. 2(b) is an oblique propagating dreamlet. The wavefront of the dreamlet atoms propagate in a limited angular range with also limited space extension. The oblique propagating dreamlet has two vertical symmetrical propagating directions because the LCB basis used for the space decomposition always has symmetrical positive and negative wavenumbers.

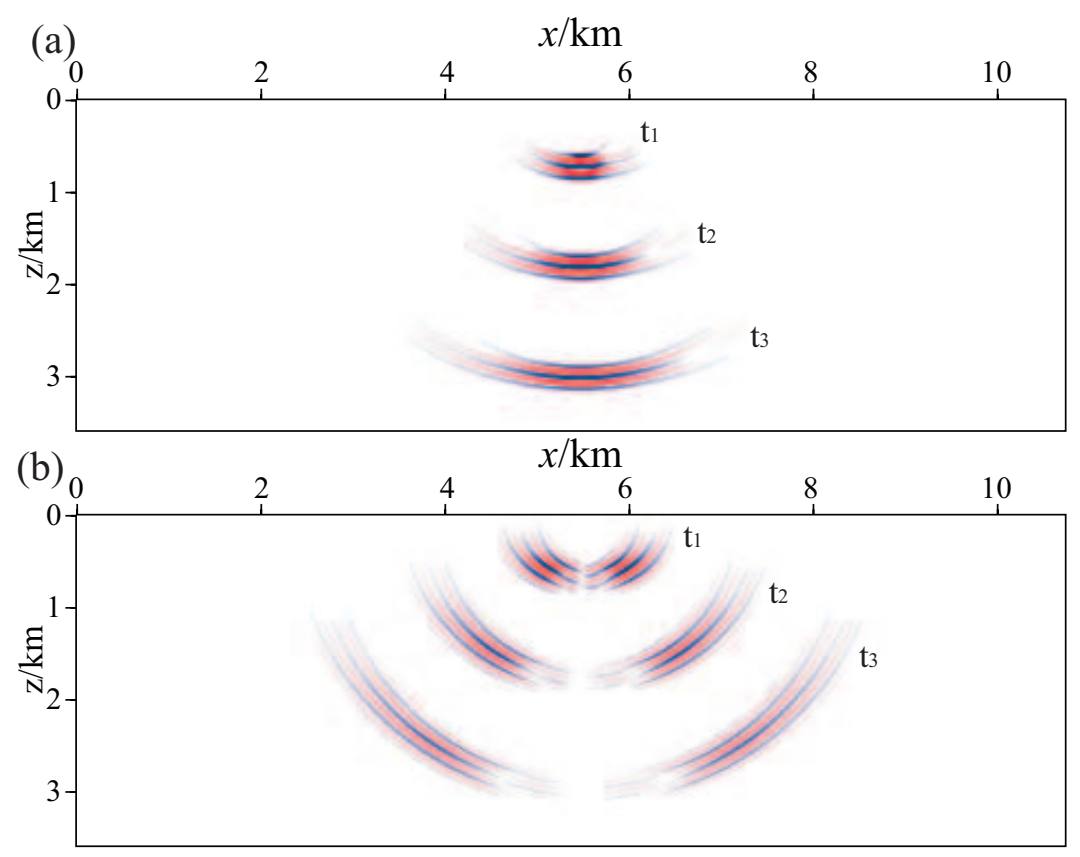

Figure 2: Single dreamlet propagation snapshots in homogenous medium: (a) Vertical propagating dreamlet; (b) Oblique propagating dreamlet. 


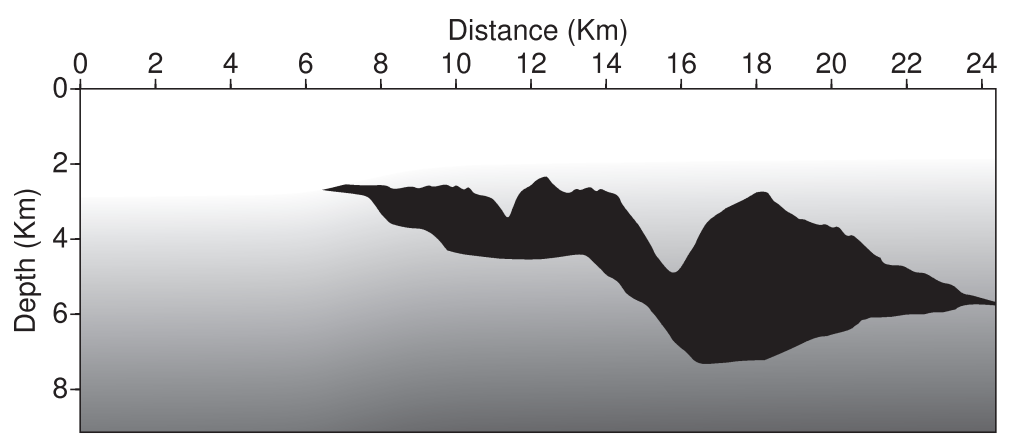

Figure 3: Sigsbee 2A velocity model for migration.

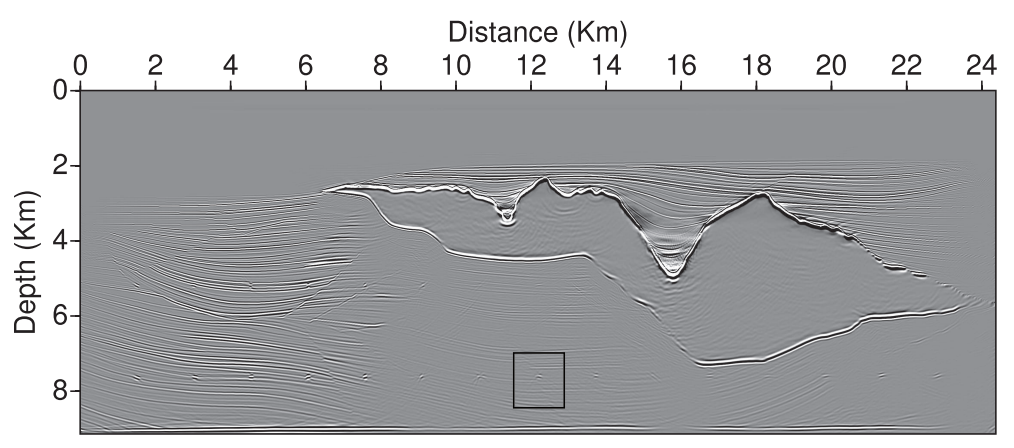

Figure 4: LEF-LCB dreamlet migration result using the migration velocity shown in Fig. 3.

The benchmark data Sigsbee2A from the SMAART Project consisting of 500 shot gathers with 348 receivers per-shot. The time sample interval is $8 \mathrm{~ms}$ with 1500 samples for each trace. The migration velocity model shown in Fig. 3 has 2133 samples in horizontal plane with an interval of 37.5 feet and 1200 samples in depth with an interval of 25 feet. The source for the migration is the ricker wavelet with a dominant frequency at $20 \mathrm{hz}$. Fig. 4 shows the LEF-LCB dreamlet migration image. The sediments, the two rows of point diffractors and the straight baseline reflector at the bottom of the model are all well imaged.

One advantage of dreamlet decomposition is its high compression ratio and the imaging in the compressed domain $[17,28]$. Fig. 5 shows the amount of dreamlet coefficient extrapolated at each depth during the migration process for the Sigsbee2A model. The dash line in the figure is for the receiver side and the solid line for the source. During migration, the average compression ratio on the receiver side is around 10 and the number of the dreamlet coefficient does not increase. The coefficient amount on the source side is increasing in shallow depth but gradually becomes saturated and still keeps a higher average compression ratio. In this salt model migration test, we apply the automatic gain control on the time series, that is, the recorded seismic data is multiplied by the square root of the recording time. The number of the velocity in the background propagator is 50 , equally spaced from the minimum to the maximum velocity in the model. 


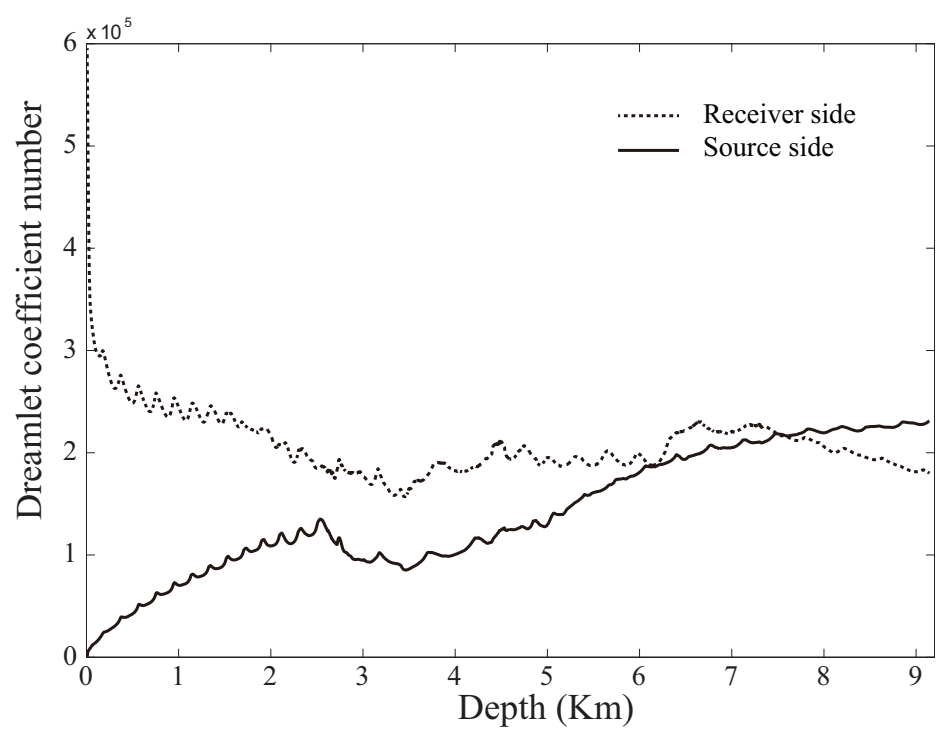

Figure 5: Coefficient amount variation during migration. The dash line is for the receiver side and the solid line for the source side wavefield.

One benefit of the localization on time using dreamlet is the easy application to target oriented migration/imaging. For a specific target area, its reflected signal on the surface is only in a few time windows in the traces at different locations. Only migrating these target related portion of data can not only accelerate the migration process but also get a better and cleaner image in the target area. For the Sigsbee2A model, suppose we focus our target on some area beneath the salt body, indicating by the box in Fig. 4 . The first arrival traveltime is first calculated and then time windows around are picked in the traces. The migration result using this portion of data is shown in Fig. 6(b). The same area with the full data set (Fig. 6(a)) is cut out from Fig. 4 for comparison. It shows clearly that the fault and reflectors in the target oriented migration are strongly imaged than that in using full data set.

The second example shows the application of LEF-LCB dreamlet migration with real marine seismic data. A 2D inline section is selected for this test. There are totally 1400 shots with 240 receivers each on this inline towing from left to right, with a source and receiver interval of 25 meter respectively. The time sampling interval is $2 \mathrm{~ms}$ with a recording length of up to 6 seconds. For the dreamlet migration, the full frequency band is used as the source wavelet (spectral amplitude is equal to 1.0 up to $140 \mathrm{~Hz}$ ). The velocity model (shown in Fig. 7) used here is obtained by ray based tomography. It contains 3100 and 800 grid points horizontally and vertically, with a cell size 12.5 by 5 meter.

For the background dreamlet propagator, a total of 100 reference velocities are calculated and equally sampled from the minimum to maximum of the velocity in this model. The threshold for the compression is $10^{-4}$ of the maximum dreamlet coefficients value at 

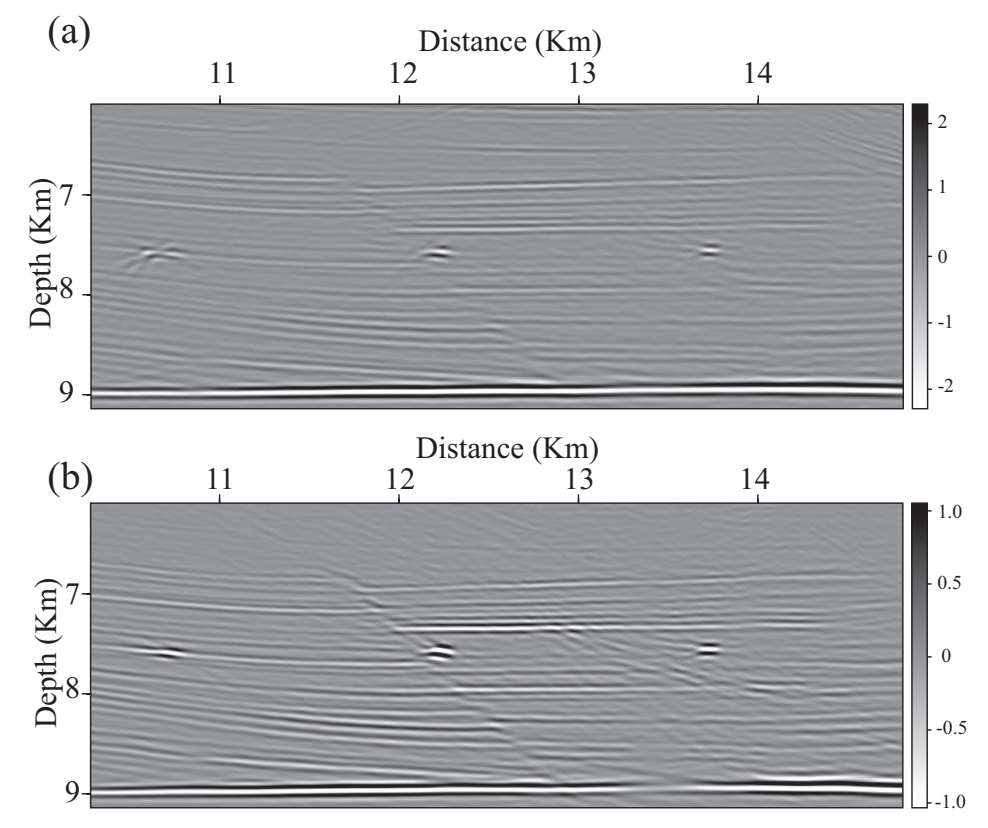

Figure 6: Comparison of the target image results use all data (a) and the target oriented data (b).

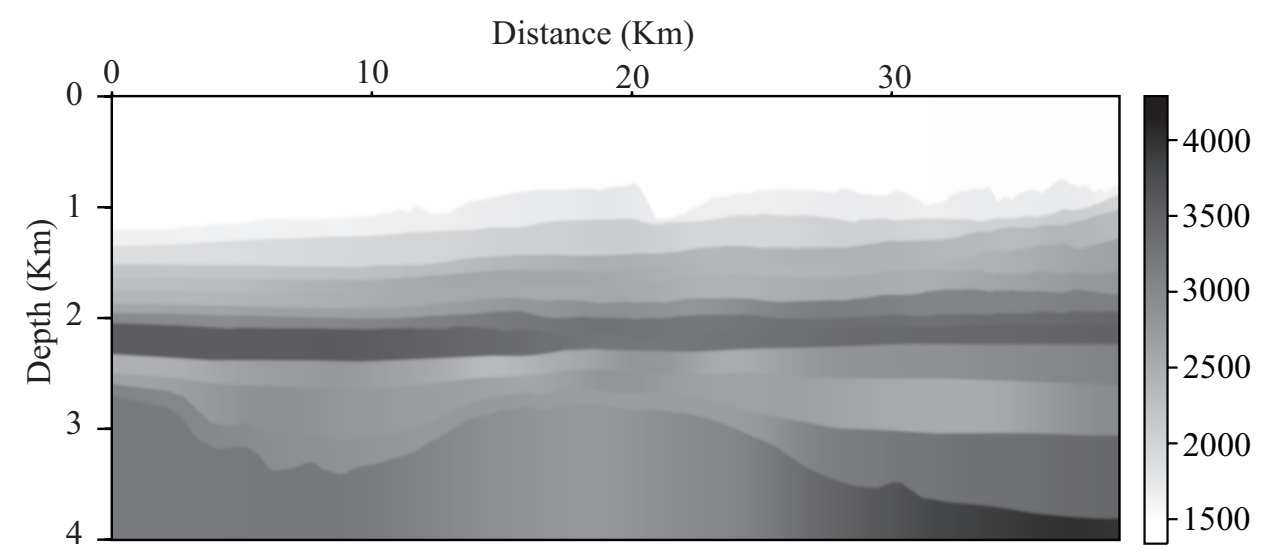

Figure 7: Migration velocity for the field marine seismic data.

each depth. Fig. 8(a) shows the LEF-LCB dreamlet migration result. Overall, the image contains broad frequency band and the fine layers are depicted clearly. The rugged sea floor is also well imaged. Due to the accurate localized wave propagation of dreamlet, the structures of buried mountain below the depth of $3 \mathrm{~km}$ is also imaged with reasonably continuous boundaries (black arrows in Fig. 8). For comparison, image from Kirchhoff and Reverse Time Migration (RTM) are shown in Figs. 8(b) and 8(c). In general, for this data set, the image quality of dreamlet migration is comparable to that of the other two methods. The image bandwidth difference is mainly due to the data filtering before mi- 

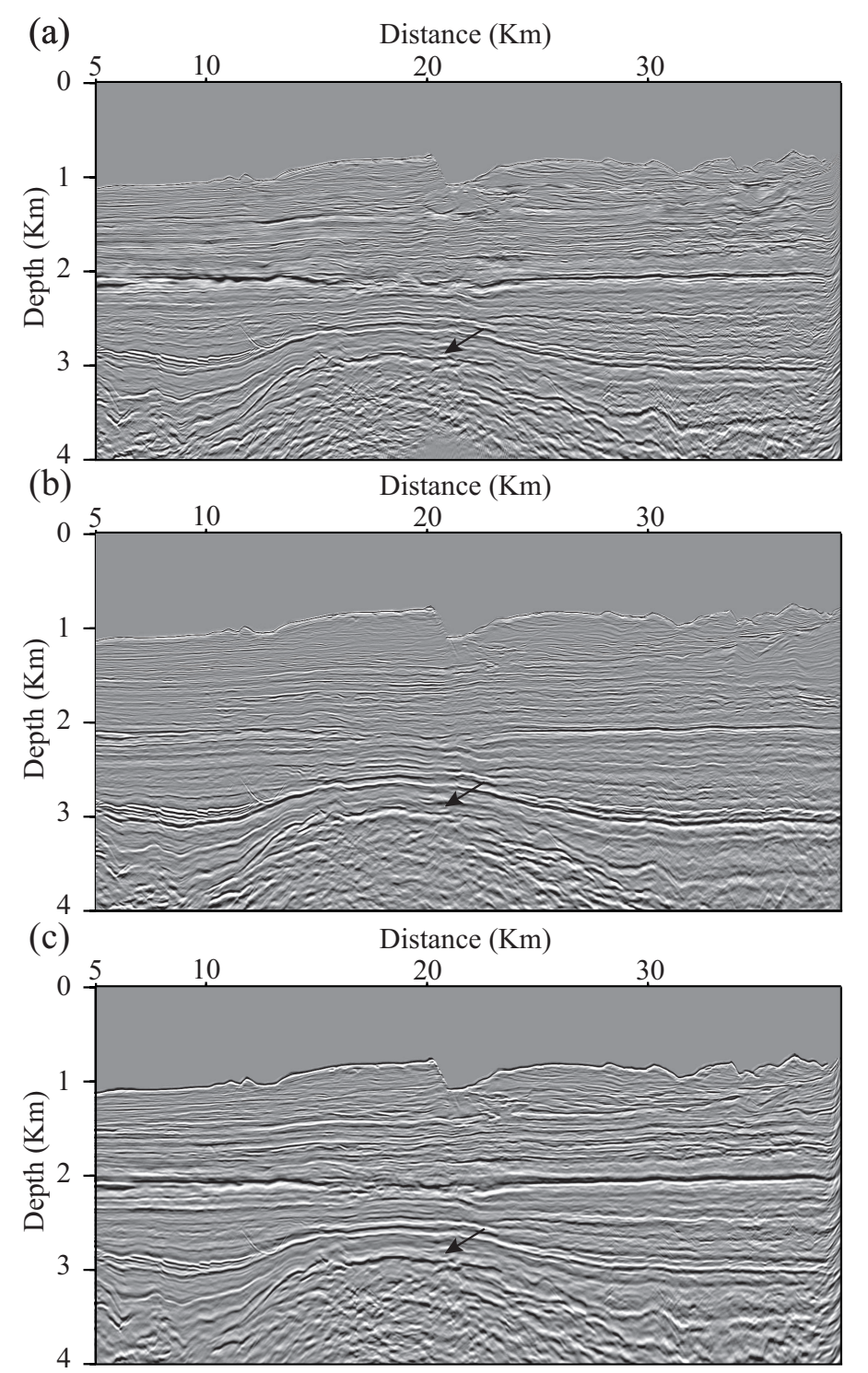

Figure 8: Marine field data migration results: (a) dreamlet migration; (b) Kirchhoff migration; (c) reverse time migration.

gration. Another difference for the images is beneath the rugged sea floor (strong lateral velocity variation), such as the horizontal location 21 and $35 \mathrm{~km}$. The images of dreamlet underneath those locations are better than that of Kirchhoff migration and slightly inferior to that of RTM. The reason is that the dreamlet propagator is approximate to the velocity lateral variations at the scale of spatial window length. The fast lateral velocity change such as the rugged sea floor, smaller spatial window length will improve the result but at the cost of migration efficiency [17]. 


\section{Conclusions and discussions}

In this paper, we first present the dreamlet migration formulation using LCB for spacedirection localization and LEF for the time frequency localization. At each depth, the data set is decomposed into dreamlets leading to a migration in the compressed domain scheme. The dreamlet propagator matrix is applied to obtain the dreamlet coefficients at the next depth level. The dreamlet propagation includes the propagation in local homogeneous reference medium followed by the local phase-screen correction. Numerical examples using Sigsbee2A model and field data example verify the high accuracy and good image quality of the method on salt model and real data. Wavefield data decomposition by dreamlet and then imaging in the dreamlet domain by dreamlet propagator have some salient features and advantages over other time-space imaging methods. First, dreamlet coefficients obtained from seismic data are very sparse due to the fact that dreamlet is a type of physical wavelet which satisfy the causality (or dispersion relation), and therefore only sit on a hyper surface in the four-dimensional time-space; second, the dreamlet propagator propagates wavefield in the dreamlet domain (compressed domain) and the sparse data can remain sparse during the imaging process, which can save computer storage substantially; third, due to the time-space localization of dreamlet representation, it is possible and easy to realize target-oriented data selection and imaging, which can not only has the flexibility of imaging a specific target of interest, but also to isolate the data portion which is responsible for the image formation. This target-oriented data and image windowing has both the high-efficiency and the high-quality of target imaging by shutting off the unnecessary data and interference. Preliminary numerical test shows the feasibility and potential application of this method to the targeted orientation migration/imaging.

\section{Acknowledgments}

This work is supported by the National Natural Science Foundation of China (41604106, 41674123, 11871392), the Fundamental Research Funds for the Center Universities (xjj2018260), the China Postdoctoral Foundation (2016M600780) and WTOPI (Wavelet Transform On Propagation and Imaging for seismic exploration) Project at University of California, Santa Cruz. We thank CNOOC for the permission to use the field example data for publication and provide the Kirchhoff migration result for comparison.

\section{References}

[1] Y. Z. Wang and R. S. Wu, Seismic data compression by an adaptive local cosine/sine transform and its effects on migration, Geophysical Prospecting, 48(2000), 1009-1031.

[2] B. F. Wang, R. S. Wu, Y. Geng and X. H. Chen, Dreamlet-based interpolation using POCS method, Journal of Applied Geophysics, 109 (2014), 256-265. 
[3] Y. Geng, R. S. Wu and J. H. Gao, Dreamlet transform applied to seismic data compression and its effects on migration, 79th Annual International Meeting, SEG, Expanded Abstracts, (2009), 3640-3644.

[4] B. F. Wang, R. S. Wu, X. H. Chen and J. Y. Li, Simultaneous seismic data interpolation and denoising with a new adaptive method based on dreamlet transform, Geophysical Journal International, 201 (2015), 1180-1192.

[5] S. H. Gray and W. P. May, Kirchhoff migration using eikonal equation traveltimes,Geophysics, 59 (1994), 810-817.

[6] J. Gazdag, Wave equation migration with the phase-shift method, Geophysics, 43 (1978), 1342-1351.

[7] P. L. Stoffa, J. T. Fokkema, R. M. de Luna Freire, W. P. Kessinger, Split-step Fourier migration, Geophysics, 55 (1990), 410-421.

[8] R. S. Wu and L. J. Huang, Scattered field calculation in heterogeneous media using phasescreen propagators, 62th Annual International Meeting, SEG, Expanded Abstracts, (1992), 1289-1292.

[9] P. Roberts, L. J. Huang, C. Burch, M. Fehler and S. Hildebrand, Prestack depth migration for complex 2D structure using phase-screen propagators, 67th Annual International Meeting, SEG, Expanded Abstracts, (1997), 1282-1285.

[10] X. B. Xie and R. S. Wu, Improve the wide angle accuracy of screen method under large contrast, 68th Annual International Meeting, SEG, Expanded Abstracts, (1998), 1811-1814.

[11] M. V. de Hoop, J. L. Rousseau and R. S. Wu, Generalization of the phase screen approximation for the scattering of acoustic waves, Wave Mothion, 31 (2000), 43-70.

[12] R. S. Wu and S. W. Jin, Windowed GSP (generalized screen propagators) migration applied to SEG-EAGE salt model data, 67th Annual International Meeting, SEG, Expanded Abstracts, (1997), 1746-1749.

[13] S. W. Jin and R. S. Wu, Depth migration with a windowed screen propagators, Journal of Seismic Exploration, 8 (1999), 27-38.

[14] R. S. Wu and L. Chen, Wave propagation and imaging using Gabor-Daubechies beamlets, in E. C. Shang, Q. Li, and T. F. Gao eds., Theoretical and Computational Acoustics 2001, World Scientific, New Jersey, (2002), 661-670.

[15] L. Chen, R. S. Wu and Y. Chen, Target-oriented beamlet migration based on GaborDaubechies frame decomposition, Geophysics, 71 (2006), S37-52.

[16] Y. Z. Wang and R. S. Wu, Beamlet prestack depth migration using local cosine basis propagator, 72th Annual International Meeting, SEG, Expanded Abstracts, (2002), 1340-1243.

[17] R. S. Wu, Y. Z. Wang and M. Q. Luo, Beamlet migration using local cosine basis, Geophysics, 73 (2008), S207-S217.

[18] Y. W. Ma, G. F. Margrave, Seismic depth imaging with the Gabor transform, Geophysics, 73 (2008), S91-S97.

[19] W. A., Imaging of the SEG/EAGE Salt Model Seismic Data Using Sparse $f$ - $x$ Finite-ImpulseResponse Wavefield Extrapolation Filters, EEE Transactions on Geoscience and Remote Sensing, 52 (2014), 2700-2714.

[20] E. J. Cands and D. L. Donoho, New Tight Frames of Curvelets and Optimal Representations of Objectives with C2 Singularities, Communications on Pure and Applied Mathematice, 57 (2002), 219-266.

[21] E. J. Cands and L. Demanet, The curvelet representation of wave propagators is optimally sparse, Communication on Pure and Applied Mathematice, 58 (2005), 1472-1528.

[22] B. F. Wang, An efficient POCS interpolation method in the frequency-space domain, IEEE 
Geoscience and Remote Sensing Letters, 13 (2016), 1384-1387.

[23] B. F. Wang, X. H. Chen, J. Y. Li and J. J. Cao, An Improved Weighted Projection Onto Convex Sets Method for Seismic Data Interpolation and Denoising, IEEE Journal of Selected Topics in Applied Earth Observations and Remote Sensing, 9 (2016), 228-235.

[24] H. Douma and M. V. de Hoop, Leading-order seismic imaging using curvelets, Geophysics, 72 (2007), S231-S248.

[25] H. Chauris and T. Nguyen, Seismic demigration/migration in the curvelet domain, Geophysics, 73 (2008), S35-S46.

[26] Y. Z. Wang, R. S. Wu and S. W. Jin, Adapted local cosine transform application to seismic data compression, The First International Symposium on Applied Geophysics, (1998), 47-50.

[27] R. S. Wu, B. Y. Wu and Y. Geng, Seismic Wave propagation and imaging using time-space wavelets, 78th Annual International Meeting, SEG, Expanded Abstracts, (2008), 2983-2987.

[28] R. S. Wu, B. Y. Wu and Y. Geng, Imaging in compressed domain using dreamlets, CPS/SEG Beijing 2009 Expanded Abstracts, (2009), ID57.

[29] P. Auscher, Remarks on the local Fourier bases, in J.J. Benedetto and M. W. Frazier eds, Wavelets, Mathematics, and Applications, CRC Press, (1994), 203-218.

[30] J. Mao and R. S. Wu, Illumination analysis using local exponential beamlets, 77th Annual International Meeting, SEG, Expanded Abstracts, (2007), 2230-2234.

[31] S. Mallat, A wavelet Tour of Signal Processing, Academic Press, second edition, 1999.

[32] I. Daubechies, S. Jaffard and J. L. Journe, A simple Wilson orthogonal basis with exponential decay, SIAM Journal of Mathematical Analysis, 22 (1991), 554-573.

[33] J. Cao and R. S. Wu, Fast acquisition aperture correction in prestack depth migration using beamelt decomposition, Geophysics, 74(2009), S67-S74.

[34] H. S. Malvar, Signal processing with lapped transforms, Artech House, 1992.

[35] R. S. Wu, Y. Geng and B. Y. Wu, Physical wavelet defined on an observation plane and the dreamlet, 81th Annual International Meeting, SEG, Expanded Abstracts, (2011), 3835-3839.

[36] Y. Geng, R. S. Wu and J. H. Gao, Gabor-frame-based Gaussian packet migration, Geophysical Prospecting, 63 (2014), 1432-1452.

[37] S. Y. Xu and S. W. Jin, Wave equation migration of turning waves, 76th Annual International Meeting, SEG, Expanded Abstracts, (2006), 2328-2332.

[38] Y. Zhang, S. Xu and G. Q. Zhang, Imaging complex salt bodies with turning-wave one-way wave equation, 76th Annual International Meeting, SEG, Expanded Abstracts, (2006), 23232327.

[39] X. F. Jia and R. S. Wu, Superwide-angle one-way wave propagator and its application in imaging steep salt flanks, Geophysics, 74 (2009), S75-S83.

[40] B. Y. Wu, R. S. Wu and J. H. Gao, Time-space localized seismic wave propagation: Dreamlet prestack depth migration, Chinese J. Geophys. (in Chinese), 55 (2012), 3105-3114.

[41] R. S. Wu, Y. Z. Wang and J. H. Gao, Beamlet migration based on local perturbation theory, 70th Annual International Meeting, SEG, Expanded Abstracts, (2000), 1008-1011. 